\title{
Spinal Unit Long Term Inpatient Management of Patients with Severe Neurological Deficit
}

\author{
E. R. Griffiths, OBE, CStJ, MB BS, FRCS, FRACS, FACRM \\ Spinal Department, Royal Perth (Rehabilitation) Hospital, Selby Street, Shenton \\ Park, Western Australia 6008.
}

\section{Summary}

Patients with the 'locked-in syndrome' are now being presented to spinal units for long term care. This is becoming a problem in spinal cord injury units, which have developed to accept spinal cord injury in the acute stage, to manage problems resulting from this, and to conduct a rehabilitation programme.

A case history is presented of a patient with Guillain-Barré's recurrent syndrome, already 3 years an inpatient in a spinal unit and with minimal recovery. Problems in care are described in a patient with total bulbar palsy, paralysis of the intercostal and of the diaphragm includes inability to swallow due to bulbar paralysis with nutrition delivered by jejunostomy. The presence of a tracheostomy, as well as the bulbar and laryngeal palsy, render vocal communication impossible.

Total care has been provided in a spinal unit for the past 3 years, with no complications occurring during his management.

Given continuation of expert care, life expectancy may be that of many years.

Is a spinal cord injury unit the proper place for long term management of cases of this nature and many similar cases described as having the 'locked-in syndrome'?

Key words: Guillain-Barré syndrome; Locked-in syndrome; Total dependence; Long term care in spinal injury unit.

\section{Locked in syndrome}

Spinal injury units have been developed to accept patients with severe neurological deficit resulting from trauma. These include management of the high tetraplegic and, when the lesion is at a high cervical level, management of respiration by tracheostomy and automatic ventilation has succeeded in preserving life for a prolonged period in many cases. These patients require nursing and medical care at a very high level, which prolongs their stay in a spinal injury unit considerably. Other patients presenting with similar problems, but not from spinal cord injury, are now presenting for care in spinal centres, and their long 


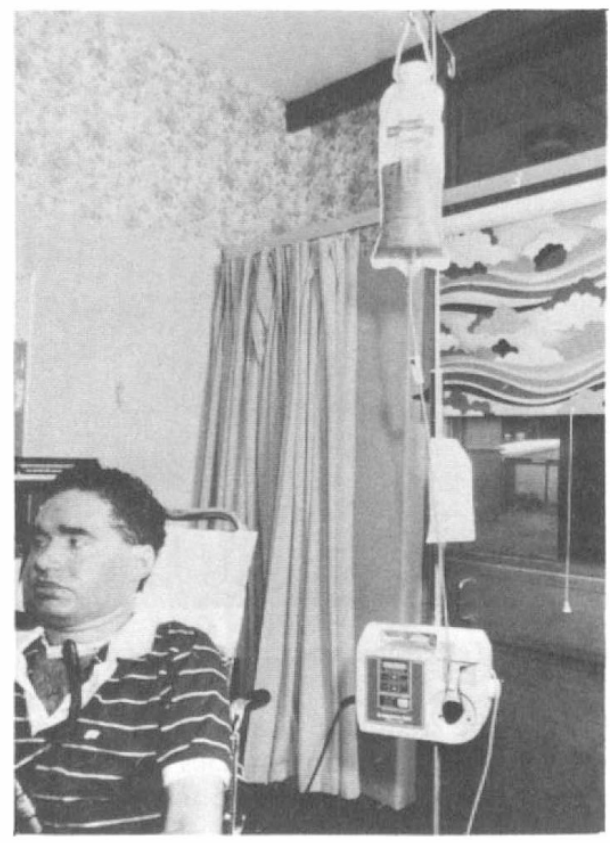

Figure 1 Nutrition via jejunostomy - special balanced fluid intake provided by dietician.

term management in a centre geared for acute management and rehabilitation may become a problem in future years.

A case history is presented where the patient in question has been an inpatient in the spinal centre in Perth for the past 3 years, with seemingly little prospect of care in other hospital departments.

\section{Case history}

This patient, a male aged 37 years, had paralysis from the Guillain-Barre's syndrome as a child. An excellent recovery was made with return to normality of all functions. In 1985, whilst working in the north west of Western Australia, some 1500 kilometres north of Perth, he realised that he was becoming weaker in his lower limbs, and remembering his previous experience as a child he drove himself to the nearest regional hospital, deteriorating gradually en route, and arrived just in time to be resuscitated and requiring the aid of a respirator.

He was transferred to the Intensive Care Department of the Royal Perth Hospital where he remained for some 7 months, with complete paralysis and maintained by a tracheostomy with assisted respiration. Minimal motor recovery occurred, with a little power in the pectoralis major and triceps and in the neck muscles. Paralysis, however, remained complete with a total bulbar palsy, paralysis of the intercostals and the diaphragm also. He was accepted for long term care in the spinal centre in January 1986, and remains an inpatient at this time, i.e., June of 1988 . His problems in management are as follows:

1. Total motor paralysis in the skeletal system with sparing of a little power in the triceps and pectoralis major in the right upper limb. Enough power remains in the neck musculature, mainly in the flexors, with which he is able to control an electric wheelchair using his chin. A specially designed module is required to support him in the sitting posture.

2. There is a complete bulbar palsy with loss of swallowing reflex and requiring suction to remove excess saliva at frequent intervals. 


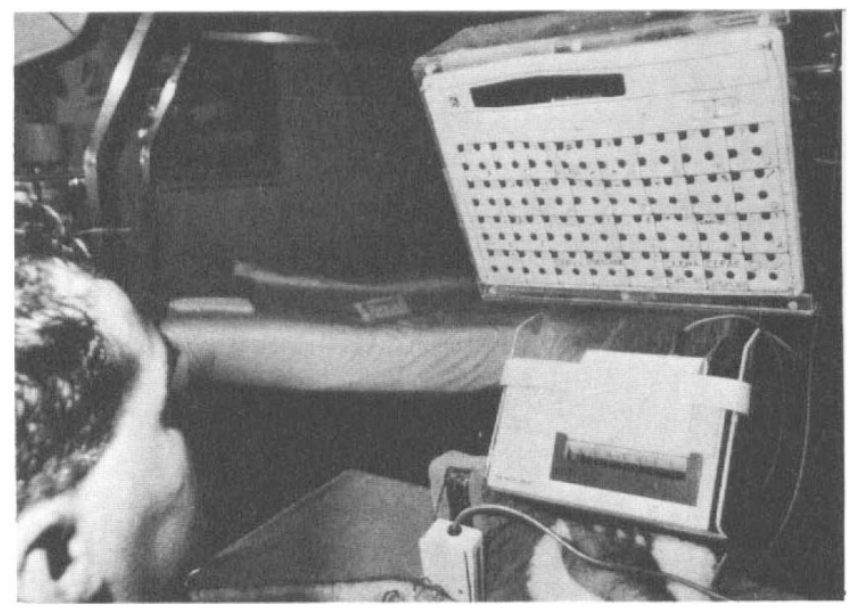

Figure 2 Communication by use of electronic keyboard and printer.

3. For the first $2 \frac{1}{2}$ years he required assisted respiration through his tracheostomy. Now with development of accessory muscles in the neck he is able to carry out 'frog breathing' and can remain off his respirator for several hours at a time, though requiring respiratory assistance at night.

4. Nutrition was initially carried out by a nasogastric tube. Eight months after onset a jejunostomy was performed, and nutrition is now continued through the jejunostomy 3 times daily, with maintenance of body weight and general condition since that time (Fig. 1.).

5. Communication has been a major problem due to the bulbar paralysis and the tracheostomy. As with a slight return of neck musculature he is able to nod, communication was initially via the nursing attendant repeating the letters of the alphabet until the right letter was reached, when a nod indicated the correct letter. Gradually by this method words and then sentences could be built up, but this was indeed very time consuming and most laborious. He is now operating an electronic communication device. The operative switch was placed adjacent to the right hand, and by a little downward pressure with the use of the triceps he can arrest the light which scans the display board, arresting it at the correct letter or number. This is then printed out, and by his own efforts he can now produce a print-out means of communication at his leisure, and pass on messages and instructions to the nursing and medical attendants (Fig. 2.).

This patient requires skilled nursing care for 24 hours a day at all times. In the past 3 years his health has been maintained throughout, and he is in a state of good nutrition, has no pressure problems, and voids urine spontaneously by suprapubic pressure. He can now, with modern technology, communicate and his intelligence is unimpaired.

There is little or no likelihood of any recovery from this major neurological defect, and no other avenue in a major hospital seems likely as an area of continuing management.

Undoubtedly the special facilities developed in the spinal injury unit have kept this man alive and well for 3 years, and may do so for a considerable period into the future.

He demonstrates very major problems in respiratory function, nutrition and communication, and in mobility.

\section{Discussion}

Is a spinal injury unit the correct place for long term management of disabilities of this nature? There are many other patients with a non-traumatic onset of a 
cerebral lesion who present in the same way, many of whom are at this time being managed in spinal centres. Is there a place for the development of special centres in a major hospital complex for long term management of cases of this nature? Should spinal injury units accept the role of management in this particular disability, in which they already have considerable expertise derived from their management of the high tetraplegic? 